\title{
A Theory for the Lower-Tropospheric Structure of the Moist Isentropic Circulation
}

\author{
FRÉDÉRIC LALIBERTÉ \\ Department of Physics, University of Toronto, Toronto, Ontario, Canada \\ TIFFANY SHAW \\ Department of Earth and Environmental Sciences, and Department of Applied Physics and Applied Mathematics, \\ Columbia University, New York, New York \\ OLIVIER PAULUIS \\ Courant Institute of Mathematical Sciences, New York University, New York, New York
}

(Manuscript received 27 February 2012, in final form 19 September 2012)

\begin{abstract}
A theoretical model describing the structure of the dry and moist isentropic circulations in the lower troposphere is derived. It decomposes the meridional flow in the troposphere into three contributions: a dry equatorward flow, a cold moist equatorward flow, and a warm moist poleward flow in the mixed layer. The model is based on observations of the meridional mass fluxes joint distribution in potential temperature and equivalent potential temperature. It updates an existing model of the dry circulation by emphasizing the role of moisture in the mixed layer. The model is used to derive an expression for the ratio of moist to dry circulation strengths and this expression is used to assess the influence of surface thermodynamics on the circulations. It predicts that the moist circulation should be between 1.5 and 2 times as strong as the dry circulation and that this relative strength should not increase indefinitely with increasing surface temperature variability. The model also yields an expression for the ratio of total meridional heat fluxes to meridional sensible heat fluxes. This expression indicates that while an increase in the total heat fluxes occurs when surface temperature variability increases (via an increase in latent heat flux), it cannot increase indefinitely. The results suggest that changes in surface thermodynamic conditions must be constrained to constrain changes in the meridional overturning circulation associated with a warming climate.
\end{abstract}

\section{Introduction}

The atmospheric circulation acts to transport energy from the equatorial regions to higher latitudes. When averaged on pressure surfaces, the circulation exhibits a three-cell structure in both hemispheres. The Ferrel cell, in midlatitudes, is associated with a poleward flow at low level and an equatorward flow at higher altitude, which corresponds to a net equatorward transport of energy. The atmospheric energy transport is poleward from equator to pole because it is dominated by large poleward energy transport by midlatitude eddies.

Corresponding author address: Frédéric Laliberté, Department of Physics, University of Toronto, 60 St. George Street, Toronto, ON M5S 1A7, Canada.

E-mail: frederic.laliberte@utoronto.ca
The relative contributions of the mean flow and eddies depends on the coordinate system in which the averaging is performed. When the circulation is averaged on dry isentropes-defined as surfaces of constant potential temperature-the atmospheric circulation appears as a single equator-to-pole cell with a poleward flow of air at high potential temperature and a return flow at lower potential temperature. The transformation from a threecell to a single-cell structure is the result of including the poleward sensible heat transport by midlatitude baroclinic eddies, which transports warm air poleward and cold air equatorward. The meridional flow near the surface is equatorward in the dry isentropic circulation and advection by this flow would suggest an equatorward transport of moisture. Since midlatitude eddies transport moisture toward the poles on dry isentropes (Schneider et al. 2006), this makes the moisture transport 
an important missing piece of the mean dry isentropic circulation.

Moisture transport by eddies can be accounted for by analyzing the circulation on moist isentropes, defined as surfaces of constant equivalent potential temperature. The circulation averaged on moist isentropes has a significantly larger mass transport than the circulation averaged on dry isentropes in midlatitudes (Pauluis et al. 2008, 2010). Laliberté et al. (2012, hereafter LSP) show that on a dry isentrope an eddy moisture transport is associated with a net poleward flow of high-equivalentpotential-temperature air balanced by a return flow of lower-equivalent-potential-temperature air. The poleward flow is associated with the warm conveyor belt of midlatitude eddies [see, e.g., Eckhardt et al. (2004) for its climatology]. In the conveyor belt, air masses are cooled as they follow slanted dry isentropes. Once they reach saturation, they thereafter ascend following a saturated moist isentrope (Emanuel 1994) to the top of the troposphere. This creates a strong coupling between moist surface air parcels and upper-tropospheric air through a combination of upright and slantwise moist convections. Czaja and Blunt (2011) demonstrate that above western boundary currents such a coupling through upright convection is common. LSP quantify the mass transport associated with the poleward flow of moist, warm air and the equatorward flow of dry, cold air, which is referred to as the moist recirculation. They demonstrate that it explains why the moist circulation has a larger total mass transport in midlatitudes than the dry circulation.

The analysis of LSP - and its interpretation in terms of moisture transport-is in apparent contradiction with the discussion of Held and Schneider (1999, hereafter HS99), where it is observed that the low-level flow on dry isentropes is mostly equatorward. HS99 argue that, at a given latitude, dynamic constraints demand that the flow should be poleward on dry isentropes that do not intersect the earth's surface. Mass conservation then implies that the return equatorward flow should occur on isentropes that intersect the earth's surface. They support their analysis with a simple statistical model to describe the structure of the low-level flow.

The primary purpose of this paper is to address this discrepancy by extending the HS99 model to include moisture transport. The result is a model where lowertropospheric mass fluxes are split into a dry, stratified layer that occupies the lower free troposphere and a moist, well-mixed layer that roughly corresponds to the boundary layer. The model presented here can qualitatively explain the difference between the midlatitude circulation on dry and moist isentropes over a range of climates. This paper begins with a short review of the
LSP analysis in section 2, followed by a review of the HS99 model and the derivation of the moist model in section 3. In section 4, the model is used to obtain an expression for the relative strength of the moist and dry isentropic circulations as well as expressions for the typical potential temperatures in the poleward and equatorward branches of the moist and dry isentropic circulations. Using these expressions, a formula for the relative importance of sensible heat in the total-sensible plus latent-meridional heat fluxes is derived. Finally, in section 5, our results are summarized and the implications for the meridional overturning circulation in a warming climate are discussed.

\section{Midlatitude overturning circulation in isentropic coordinates}

We begin by reviewing some recent results on the differences between the circulation averaged on dry isentropes and the circulation averaged on moist isentropes. To illustrate how the two circulations differ, it is useful to describe the properties of air parcels crossing a given latitude by obtaining a relationship between meridional motions and thermodynamic quantities. A succinct representation of this relationship is the seasonalmean meridional mass flux joint $\left(\theta, \theta_{e}\right)$ distribution

$$
M\left(\phi, \theta, \theta_{e}\right)=2 \pi a \cos \phi\left[\int_{0}^{p_{\text {surf }}} v \delta\left(\theta-\theta^{\prime}\right) \delta\left(\theta_{e}-\theta_{e}^{\prime}\right) \frac{d p}{g}\right],
$$

where the square brackets represent a zonal and temporal mean, $\delta(\cdot)$ corresponds to the Dirac delta function, $\theta$ is the dry potential temperature, and $\theta_{e}$ is the equivalent potential temperature. The two potential temperatures $\theta$ and $\theta_{e}$ are related by

$$
\theta_{e} \approx \theta \exp \left(\frac{L_{v 0}}{c_{p} T} q_{T}\right) \approx \theta+\kappa \frac{L_{v 0}}{R_{v}}\left(p / p_{0}\right)^{\kappa} \mathcal{H} e_{s}\left\{\theta\left(p / p_{0}\right)^{\kappa}\right\} / p,
$$

where $q_{T}$ is the specific water content, $e_{S}(T)$ is the saturation vapor pressure, $L_{v 0}$ is the latent heat of vaporization, $c_{p}$ is the specific heat at constant pressure, $\kappa$ is the adiabatic expansion coefficient, $\mathcal{H}$ is the relative humidity, and $R_{v}$ is the gas constant for water vapor. The surface pressure $p_{\text {surf }}$, the meridional velocity $v$, and the primed potential temperatures are obtained from 6-hourly observations. A list of the remaining parameters is provided in Table 1. The meridional mass flux joint distribution is computed using the numerical method described by LSP. This method guarantees that meridional 
TABLE 1. List of parameters.

\begin{tabular}{|c|c|c|}
\hline Variables & Value & Descriptions \\
\hline$\lambda, \phi, p, t$ & & Longitude, latitude, pressure, time \\
\hline$g, a$ & & Gravitational acceleration, the earth's radius \\
\hline $\mathcal{H}$ & & Relative humidity \\
\hline$e_{s}(T)$ & & Saturation water vapor pressure, given by the Clausius-Clapeyron relation \\
\hline $\mathbb{H}(\cdot)$ & & Heaviside function; 0 for a negative argument and 1 otherwise \\
\hline$\delta(\cdot)$ & & Dirac delta function \\
\hline$\theta, \theta_{e}$ & & Dry potential temperature, equivalent potential temperature \\
\hline$L_{v 0}$ & $2.5 \times 10^{6} \mathrm{~J}$ & Latent heat of vaporization at $0^{\circ} \mathrm{C}$ \\
\hline$R_{v}$ & $461.5 \mathrm{~J} \mathrm{~K}^{-1}$ & Water vapor gas constant \\
\hline$c_{p}$ & $1615.25 \mathrm{~J} \mathrm{~K}^{-1}$ & Specific heat of dry air \\
\hline$\kappa$ & $2 / 7$ & Adiabatic expansion coefficient \\
\hline$T_{0}$ & $273.15 \mathrm{~K}$ & Reference temperature \\
\hline$p_{0}$ & $1013 \mathrm{hPa}$ & Reference surface pressure \\
\hline
\end{tabular}

fluxes of dry and equivalent potential temperatures can be recovered exactly from the joint distribution. The observations presented in this paper are based on the 40yr European Centre for Medium-Range Weather Forecasts (ECMWF) Re-Analysis (ERA-40) dataset (Uppala et al. 2005) over the period 1981-2000. It was obtained from the National Center for Atmospheric Research Data Support Section, dataset ds127.2.

In Fig. 1, we show the meridional mass flux joint distribution, at $35^{\circ} \mathrm{N}$ during December-February (DJF), as in LSP (their Fig. 2). The thick dashed horizontal line indicates the mean surface dry potential temperature $\overline{\theta_{s}} \approx 288 \mathrm{~K}$. The 95 th percentile of the surface potential temperature is marked by the thick black line in Fig. 1, corresponding to $\theta_{I} \approx 304 \mathrm{~K}$. As noted in Pauluis et al. (2008, 2010) the poleward flow (red shading) occurs preferentially at high values of $\theta_{e}$, while the equatorward flow (blue shading) occurs at low values of $\theta_{e}$. The poleward and equatorward mass fluxes are better separated by their equivalent potential temperature value than their potential temperature value. This partly explains why the mass transport on moist isentropes is larger than the mass transport on dry isentropes. HS99 argue that the mass fluxes are primarily poleward above $\theta_{I}$ because of dynamical constraints related to the downgradient flux of potential vorticity in the free troposphere. This constraint demands that the equatorward return flow must occur within dry isentropes that intersect the earth's surface (i.e., at potential temperatures lower than or equal to $\left.\theta_{I}\right)$. In the joint distribution, the mass fluxes on dry isentropes below $\overline{\theta_{s}}$ (e.g., for $\theta<\overline{\theta_{s}}$ ) are equatorward, which is in agreement with HS99. However, while the net mass transport on dry isentropes between 288 and $304 \mathrm{~K}$ is indeed toward the equator (the sum across $\theta_{e}$ is negative; see LSP, their Fig. 2, left), Fig. 1 also reveals the presence of large poleward mass fluxes at high $\theta_{e}$, corresponding to relatively moist air.
As air parcels can move up and down while conserving their $\theta$ and $\theta_{e}$ value, it is not possible to equate a position in the joint-distribution $\theta-\theta_{e}$ plane to a specific vertical location. Therefore, in order to gain more insight on the vertical structure of the flow, Fig. 1 also labels the combinations of $\theta$ and $\theta_{e}$ for which air parcels are saturated at a total pressure of 1000,850 , and $700 \mathrm{hPa}$. As the degree of saturation decreases with height, any air parcels in the $\theta-\theta_{e}$ plane that lie below the 850 -hPa curve would be supersaturated if they were brought to a pressure of $850 \mathrm{hPa}$ or lower. Since supersaturation is rarely present in the atmosphere (and is absent from the reanalysis data used here), parcels that lie below the 850-hPa curve in Fig. 1 can only be found at a pressure

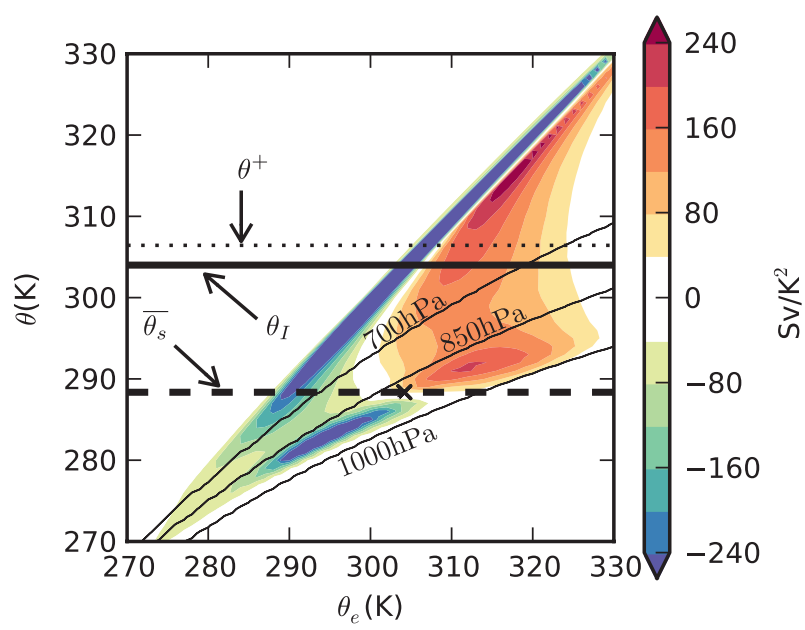

FIG. 1. Mass flux joint distribution for DJF at $\phi=35^{\circ} \mathrm{N}$. The thin black lines show the saturation curve at different pressure levels. The thick black line indicates the cutoff $\theta_{I}$ and the thick dashed black line shows the mean surface dry potential temperature. The black dotted line shows the typical potential temperature in the poleward branch of the dry circulation. The cross marks the mean surface equivalent potential temperature. 
higher or equal to $850 \mathrm{hPa}$. This interpretation of the joint distribution suggests that a large fraction of air parcels found on dry isentropes intercepting the surface (i.e., for $\theta<\theta_{I}$ ) are physically located in the lower troposphere. The saturation curves effectively split the joint distribution between a boundary layer-like region below the $850-\mathrm{hPa}$ curve and a free-tropospheric region above. The mass fluxes within this region correspond approximately to air parcels with a relative humidity of $60 \%$ at the earth's surface, and comprise two peaks of opposite signs and similar strength, separated by the $\theta=\overline{\theta_{s}}$ line. Above this line, mass fluxes are poleward and, below, they are equatorward.

In summary, the joint distribution reveals that mass fluxes in the lower troposphere can be decomposed into three main components: 1 ) an equatorward flow of cold, moist air associated with potential temperatures less than the mean surface value $\theta<\overline{\theta_{s}} ; 2$ ) an equatorward flow of dry air with $\overline{\theta_{s}}<\theta<\theta_{I}$; and 3) a poleward flow of warm, moist air occurring at similar potential temperatures, with $\overline{\theta_{s}}<\theta<\theta_{I}$, but higher equivalent potential temperature. In the next section, we present a theory that describes these three different flows. Note that the equatorward flow of dry air with $\overline{\theta_{s}}<\theta<\theta_{I}$ extends to potential temperatures higher than $\theta_{I}$. This free-tropospheric flow was described in LSP and was attributed to the moist recirculation. It is not directly accounted for by the theory developed next.

\section{Model of the near-surface flow}

\section{a. Dry model}

In this section, we review the dry kinematic model of HS99, summarized as a schematic in Fig. 2, and subsequently extend the model to include moisture transport. The HS99 model describes the lower-tropospheric branch of the dry isentropic circulation. It represents a simplified model of the warm poleward flow and cold equatorward flow seen in dry isentropic coordinates. The poleward and equatorward flows are assumed to occur within different isentropic layers. The equatorward flow occurs in the "surface layer" defined as $\theta \leq \theta_{I}$. The poleward flow occurs for $\theta>\theta_{I}$. Here, as in HS99, we use the probability distribution of dry potential temperatures at the earth's surface $\Pi\left(\theta_{s}\right)$ to define $\theta_{I}$ as the 95th percentile of the distribution. We follow HS99 and assume that $\Pi\left(\theta_{s}\right)$ is Gaussian, which is generally a good assumption in midlatitudes. In the appendix, we discuss this assumption and we present several integral identities involving the Gaussian distribution that will prove useful in the derivation of our model.

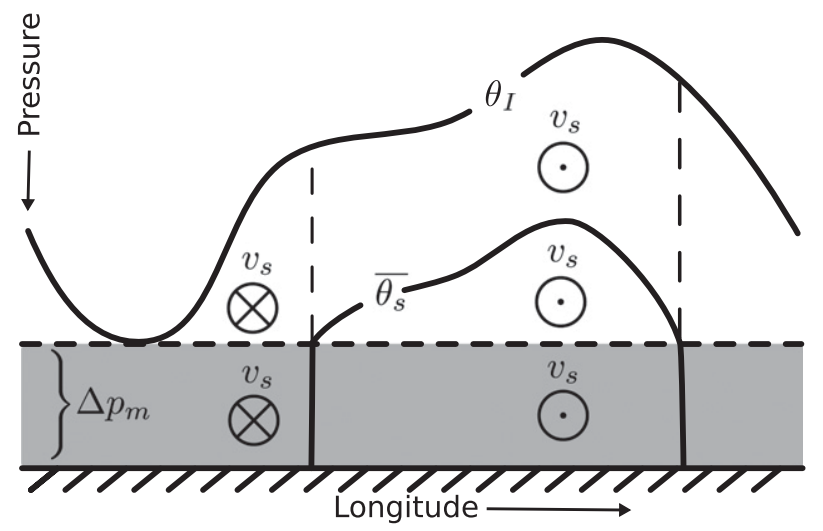

FIG. 2. Diagram for the surface- and mixed-layer model of section 3. The direction of $v_{s}$ is equatorward (circled dot) in columns where surface $\theta_{s}$ is below $\overline{\theta_{s}}$ and is poleward (circled cross) in columns where surface $\theta_{s}$ is above $\overline{\theta_{s}}$. The shaded region, corresponding to the mixed layer, is assumed moist in the new model presented in this paper.

In HS99, temporal and longitudinal variations in meridional velocity within the surface layer are assumed to be vertically uniform and proportional to deviations from the mean dry potential temperature at the earth's surface; for example,

$$
v_{s}(\lambda, \phi, t)=\alpha\left\{\theta_{s}(\lambda, \phi, t)-\overline{\theta_{s}}\right\},
$$

where $v_{s}$ is the meridional velocity in the surface layer, $\theta_{s}(\lambda, \phi, t)$ is the dry potential temperature at the earth's surface. The factor $\alpha$ is positive in the Northern Hemisphere $(\mathrm{NH})$ and negative in the Southern Hemisphere (SH). The conclusions resulting from the model do not depend on $\alpha$. This linear relation is the simplest expression that guarantees that, on average, warm air flows poleward and cold air flows equatorward and is depicted in Fig. 2 with equatorward meridional velocities below $\overline{\theta_{s}}$ and poleward meridional velocities between $\overline{\theta_{s}}$ and $\theta_{I}$.

For simplicity, HS99 neglect vertical variations in static stability and assign a constant vertical stratification equal to $H^{-1}=g \Delta \theta_{s} / \Delta p_{s}$. Here, we depart slightly from their derivation by setting $\Delta p_{s}$ equal to a constant and $\Delta \theta_{s}$ equal to the standard deviation of dry surface potential temperature. This makes the surface layer stratification directly related to the variance in surface potential temperature. The static stability defines the mass of an isentrope within the surface layer and, when it is combined with Eq. (3), we can compute the meridional mass flux:

$$
H v_{s}(\lambda, \phi, t)=H \alpha\left\{\theta_{s}(\lambda, \phi, t)-\overline{\theta_{s}}\right\}
$$

for a given isentropic layer. If the isentropic layer is below the ground [i.e., $\theta<\theta_{s}(\lambda, \phi, t)$ ] we set this mass 
flux to zero. Without this condition, Eq. (4) would depend only on $\theta_{s}(\lambda, \phi, t)$ and would be independent of $\theta$. The average mass flux on a fixed isentropic layer $\theta$ for all time and longitudes can be represented by a closed-form expression by using the probability distribution $\Pi\left(\theta_{s}\right)$. This is accomplished by first observing that a given $\theta_{s}$ occurs a fraction $\Pi\left(\theta_{s}\right) d \theta_{s}$ of the time along the latitude band. When the potential temperature at the earth's surface is equal to $\theta_{s}$, the mass flux on all isentropes $\theta$ within the surface layer and with $\theta_{I}>\theta>\theta_{s}$ is given by Eq. (4). Thus, on any isentrope $\theta$ such that $\theta_{I}>\theta>\theta_{s}$ the contribution from $\theta_{s}$ to the time and zonal-mean isentropic mass flux will be equal to expression (4) times $\Pi(\theta) d \theta_{s}$, the fraction of the time this $\theta_{s}$ occurs. If $\theta_{s}>\theta$, this contribution will be zero. Summing the contributions from all surface potential temperatures, we obtain the mass fluxes in the surface layer:

$$
\langle\rho v\rangle_{s}(\theta)=\int_{0}^{\theta} H \alpha\left(\theta_{s}-\overline{\theta_{s}}\right) \Pi\left(\theta_{s}\right) d \theta_{s}=-\frac{1}{g} \Delta p_{s} \alpha \Delta \theta_{s} \Pi(\theta),
$$

where we have made use of the Gaussian assumption to obtain the second equality. According to this formulation, an isentrope more than a few standard deviations away from $\overline{\theta_{s}}$ contributes little to the average surfacelayer mass flux. Note that $\langle\rho v\rangle_{\mathrm{s}}$ peaks at $\theta=\overline{\theta_{s}}$ and is equatorward for all $\theta$. In Fig. 1, this mass flux corresponds to the equatorward mass fluxes along the main diagonal. In contrast to the theory, the mass fluxes in the joint distribution of Fig. 1 do not peak at $\theta=\overline{\theta_{s}}$ and remain large at higher $\theta$ values. This is because the joint distribution includes free-tropospheric mass fluxes generated by the moist recirculation. As shown in LSP, the moist recirculation is associated with a dry equatorward flow balancing the poleward flow of warm, moist air undergoing large-scale moist ascents in midlatitude eddies. This equatorward flow is therefore unrelated to a surface dynamic balance and, consequently, is not included in our model.

In addition to the surface layer, HS99 specify a mixed layer with mass thickness $\Delta p_{m} / g$. Physically, the mixed layer corresponds roughly to the boundary layer and is characterized in their model by vertically uniform dynamics. Within the mixed layer, HS99 assume a vertically uniform dry potential temperature equal to the earth's surface temperature and a vertically uniform velocity given by Eq. (3). In Fig. 1, the mixed layer would lie below the $850-\mathrm{hPa}$ saturation curve and in Fig. 2 it is identified by gray shading. The mass flux associated with a mixed layer of vertically uniform dry potential temperature will thus be equal to its mass times its velocity:

$$
\frac{1}{g} \Delta p_{m} v_{s}(\lambda, \phi, t)=\frac{1}{g} \Delta p_{m} \alpha\left\{\theta_{s}(\lambda, \phi, t)-\overline{\theta_{s}}\right\} .
$$

As for the surface layer, we average Eq. (6) over times and longitudes on a fixed isentropic layer $\theta$. To do so, we imagine an isentropic layer of finite thickness bounded by $\theta$ and $\theta+\delta \theta$. This layer will be found in the mixed layer only when $\theta<\theta_{s}(\lambda, \phi, t)<\theta+\delta \theta$. If $\delta \theta$ is infinitesimally small, this will happen a fraction $\Pi\left(\theta_{s}\right) \delta \theta$ of the time. When the isentropic layer is not found within the mixed layer, there is no mixed-layer contribution to the isentropic layer's mean mass flux. The mixed layer's contribution to the mass flux on the isentrope $\theta$ will thus be

$$
\langle\rho v\rangle_{m}(\theta)=\frac{1}{g} \Delta p_{m} \alpha\left(\theta-\overline{\theta_{s}}\right) \Pi(\theta)
$$

after dividing by $\delta \theta$ on both sides.

We obtain the net mass fluxes on dry isentropes $\langle\rho v\rangle$ by adding the contributions from the mixed and surface layers:

$$
\begin{aligned}
\langle\rho v\rangle & =\langle\rho v\rangle_{s}+\langle\rho v\rangle_{m}, \\
& =\left\{\left(\theta-\overline{\theta_{s}}\right)-\frac{\Delta p_{s}}{\Delta p_{m}} \Delta \theta_{s}\right\} \frac{\Delta p_{m} \alpha}{g} \Pi(\theta) .
\end{aligned}
$$

This expression describes an equatorward flow for $\theta<\overline{\theta_{s}}$ but not necessarily for $\theta \geq \overline{\theta_{s}}$ since the first term becomes poleward and the second term remains equatorward. The net mass flux on an isentrope $\theta \geq \overline{\theta_{s}}$ can thus be either equatorward or poleward, depending on the ratio $\Delta p_{s} / \Delta p_{m}$.

\section{b. Moist model}

The HS99 model captures the warm poleward and cold equatorward mass transport included in the dry isentropic circulation but does not include any moisture transport. As it was observed in the joint distribution of Fig. 1, the surface layer is mostly dry and it contributes little to the net meridional moisture transport. The mixed layer, on the other hand, is moist with mass fluxes occurring at about $60 \%$ relative humidity. To extend the HS99 model to include moisture transports, it would therefore be sufficient to alter their definition of the mixed layer. This is accomplished by linearizing Eq. (2) about $\overline{\theta_{s}}$ at each latitude and setting the pressure equal to the surface value $p_{0}=1013 \mathrm{hPa}$; for example,

$$
\theta_{\text {em }}(\theta) \approx \overline{\theta_{e s}}+L\left(\theta-\overline{\theta_{s}}\right),
$$


where $L=1+\kappa\left\{L_{v 0} /\left(R_{v} \overline{\theta_{s}}\right)\right\}^{2}\left\{e_{s}\left(\overline{\theta_{s}}\right) / p_{0}\right\}$ and the mean surface equivalent potential temperature is $\overline{\theta_{e s}}=\overline{\theta_{s}}+$ $\kappa L_{v 0} / R_{v} \mathcal{H}\left\{e_{s}\left(\overline{\theta_{s}}\right) / p_{0}\right\}$. The terms $\kappa, L_{v 0}, R_{v}$, and $p_{0}$ can be found in Table 1 . With $\mathcal{H}=60 \%$, and $\overline{\theta_{s}}=290 \mathrm{~K}$, the linearization coefficient has a value $L=2.13$. In this expression, $L$ represents an upper-bound estimate because we are neglecting pressure variations in the mixed layer.

As discussed in section 2, the mass flux on dry isentropes results in significant cancellations of equatorward and poleward mass fluxes for $\theta>\overline{\theta_{s}}$. The cancellations arise from opposing surface-layer and mixed-layer mass fluxes occurring at the same potential temperature, thus producing no net sensible heat fluxes. The difference in moisture content between the two layers should however generate latent heat fluxes that are unaccounted for in the HS99 theory. To avoid the cancellations and to properly account for latent heat fluxes, we use a method inspired by the analysis of LSP. We decompose Eq. (8) into equatorward and poleward mass fluxes before combining the surface-layer and the mixed-layer contributions. This avoids the cancellation and preserves the full thermodynamic structure of the lower-tropospheric circulation. We apply this method to the HS99 model by first isolating the mass fluxes within the mixed layer and on isentropes below $\overline{\theta_{s}}$. We then add these mass fluxes to the surface layer's contribution to form the equatorward directional fluxes $\langle\rho v\rangle^{-}$. Finally, we attribute the remaining mixedlayer mass fluxes to the poleward directional fluxes $\langle\rho v\rangle^{+}$:

$$
\begin{aligned}
\langle\rho v\rangle^{-} & =\min \left(\langle\rho v\rangle_{m}, 0\right)+\langle\rho v\rangle_{s} \\
& =-\left(\alpha \Delta p_{m} / g\right)\left\{\max \left(\overline{\theta_{s}}-\theta, 0\right)+\frac{\Delta p_{s}}{\Delta p_{m}} \Delta \theta_{s}\right\} \Pi(\theta),
\end{aligned}
$$

$$
\langle\rho v\rangle^{+}=\max \left(\langle\rho v\rangle_{m}, 0\right)=\left(\alpha \Delta p_{m} / g\right) \max \left(\theta-\overline{\theta_{s}}, 0\right) \Pi(\theta),
$$

where we have assumed that we are in the NH. The directional mass fluxes in Eqs. (9b)-(9c) are consistent with the joint distribution. On isentropes below the mean temperature at the earth's surface, $\theta<\overline{\theta_{s}}$, poleward mass fluxes are zero and the equatorward mass fluxes are primarily associated with cold, moist air in the mixed layer. On warmer isentropes, for $\overline{\theta_{s}}<\theta<\theta_{I}$, there is both an equatorward flow, associated with dry air located above the mixed layer, and a poleward flow of warm, moist air within the mixed layer. Physically, the equatorward mass fluxes can be interpreted as a flow of dry air in the free troposphere that is injected into the planetary boundary layer. It is then gradually moistened as it moves closer to the equator, with the poleward flow corresponding to warmer and moister air parcels for the subtropical regions.

\section{Implications for dry and moist isentropic circulations}

\section{a. Circulation strengths}

In this section, we use our model of the near-surface flow to study the strengths of the circulations on moist and dry isentropes. In general, the moist and dry circulations can be recovered directly from the mass flux joint distribution; that is,

$$
\begin{gathered}
\Psi_{\theta}(\phi, \theta)=\int_{0}^{\theta} \int_{0}^{\infty} M\left(\phi, \theta, \theta_{e}\right) d \theta_{e} d \theta \\
\Psi_{\theta_{e}}\left(\phi, \theta_{e}\right)=\int_{0}^{\theta_{e}} \int_{0}^{\infty} M\left(\phi, \theta, \theta_{e}\right) d \theta d \theta_{e} .
\end{gathered}
$$

Following Pauluis et al. (2010), we quantify the strength of the circulations using the total mass transports $\Delta \Psi_{\theta}=\max _{\theta}\left\{\Psi_{\theta}(\phi, \theta)\right\}-\min _{\theta}\left\{\Psi_{\theta}(\phi, \theta)\right\}$ and $\Delta \Psi_{\theta_{e}}=$ $\max _{\theta}\left\{\Psi_{\theta_{e}}\left(\phi, \theta_{e}\right)\right\}-\min _{\theta}\left\{\Psi_{\theta_{e}}\left(\phi, \theta_{e}\right)\right\}$.

In LSP, it was demonstrated that the equatorward and poleward directional fluxes can be used to approximate the moist isentropic circulation. The approximate circulation showed that moist poleward fluxes played a key role in setting the strength of the moist isentropic circulation. Here, we use the directional fluxes from the moist model in Eq. (9) to obtain a similar result. We compute the ratio of the moist and dry circulation strengths and use it to show how the relative depth of the mixed- and surface-layer controls the meridional overturning. To compute the ratio of circulation strengths, we first derive an expression for the dry circulation strength. The dry circulation strength is equal to the maximum of the dry isentropic streamfunction and this maximum is attained when the net mass fluxes on dry isentropes $\langle\rho v\rangle$ vanish. From Eqs. (8) and (9), we see that this occurs at $\theta_{m}=\overline{\theta_{s}}+\Delta \theta_{s}\left(\Delta p_{s} / \Delta p_{m}\right)$. The dry circulation strength will thus be equal to the streamfunction value at $\theta_{m}: \Delta \Psi_{\theta}=\int_{0}^{\theta_{m}}\langle\rho v\rangle d \theta$.

To obtain a similar expression for the moist circulation, we assume that the moist circulation strength is equal to the net equatorward mass fluxes in the surface layer. This implies that most of the eddy water vapor transport is confined to the mixed layer. Under these assumptions, the model indicates that the ratio of the circulation strengths depends on the ratio of the depths of the surface and mixed layers:

$$
\begin{aligned}
\frac{\Delta \Psi_{\theta_{e}}}{\Delta \Psi_{\theta}} & =\int_{0}^{\infty}\langle\rho v\rangle^{-} d \theta / \int_{0}^{\theta_{m}}\langle\rho v\rangle d \theta, \\
& =2\left\{e^{-\Delta p_{s}^{2} / \Delta p_{m}^{2}}+\operatorname{erf}\left(\frac{\Delta p_{s}}{\Delta p_{m}}\right)\right\}^{-1},
\end{aligned}
$$




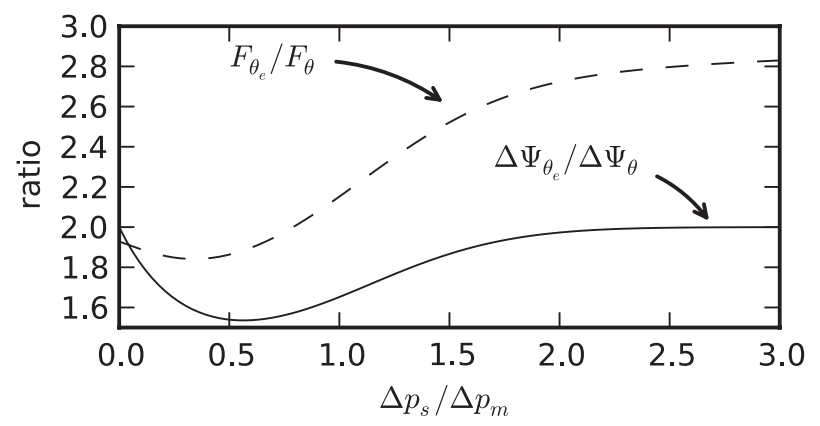

FIG. 3. The solid line relates the ratio of moist to dry circulation strengths to the ratio of surface- to mixed-layer depth according to Eq. (11). The dashed line shows the ratio of moist to dry entropy fluxes as predicted by Eq. (15) with $\overline{\theta_{e s}}, \Delta \theta_{e s}, \overline{\theta_{s}}$, and $\Delta \theta_{s}$ taken from observations at $35^{\circ} \mathrm{N}$.

where $\operatorname{erf}(\cdot)$ represents the error function. Note that this expression does not depend on $\alpha$, the parameter that sets the magnitude of surface meridional velocity. The ratio of circulation strengths only depends on the characteristics of the mass flux joint distribution, which can be obtained from reanalysis data.

Figure 3 shows the ratio $\Delta \Psi_{\theta_{e}} / \Delta \Psi_{\theta}$ using Eq. (11) for a range of $\Delta p_{s} / \Delta p_{m}$ values. It exhibits a minimum of $\Delta \Psi_{\theta_{e}} / \Delta \Psi_{\theta} \approx 1.55$ at $\Delta p_{s} / \Delta p_{m} \approx 0.5$ and asymptotically approaches 2 for large $\Delta p_{s} / \Delta p_{m}$. In HS99, the authors suggest $\Delta p_{s} \gtrsim \Delta p_{m}$, which implies a ratio $2 \gtrsim \Delta \Psi_{\theta_{e}} / \Delta \Psi_{\theta} \gtrsim 1.7$, which is in accordance with the results of Pauluis et al. (2010). Our model predicts that the moist circulation should be about twice as strong as the dry circulation, assuming that the probability distribution function of the surface dry potential temperature $\Pi\left(\theta_{s}\right)$ is Gaussian and moisture is confined to the mixed layer. We show in Fig. 4 the ratio of the moist and dry isentropic circulations for DJF, June-August (JJA), and the annual mean for 1981-2000 from the ERA-40 dataset. In midlatitudes, the ratios are in qualitative agreement with the model predictions. In LSP, it is observed that summer moist dynamics is very different from winter moist dynamics, especially in the NH. It is shown in the right panels of LSP's Fig. 6 that the 90th percentile of $\theta_{s}$-roughly corresponding to $\theta_{I}$ here-is equal to $310 \mathrm{~K}$ while the median $\theta_{s}$ is equal to $300 \mathrm{~K}$ between $30^{\circ}$ and $40^{\circ} \mathrm{N}$. This indicates that during the $\mathrm{NH}$ summer the distribution $\Pi\left(\theta_{s}\right)$ is skewed toward high $\theta_{s}$. To a lesser extent, the same is true during the SH summer. The theory proposed here, because it relies on $\Pi\left(\theta_{s}\right)$ being Gaussian, should therefore not be applicable to the summer season. Indeed, the ratios obtained for the summer season are much larger than expected from the theory (not shown) and were excluded from our analysis.

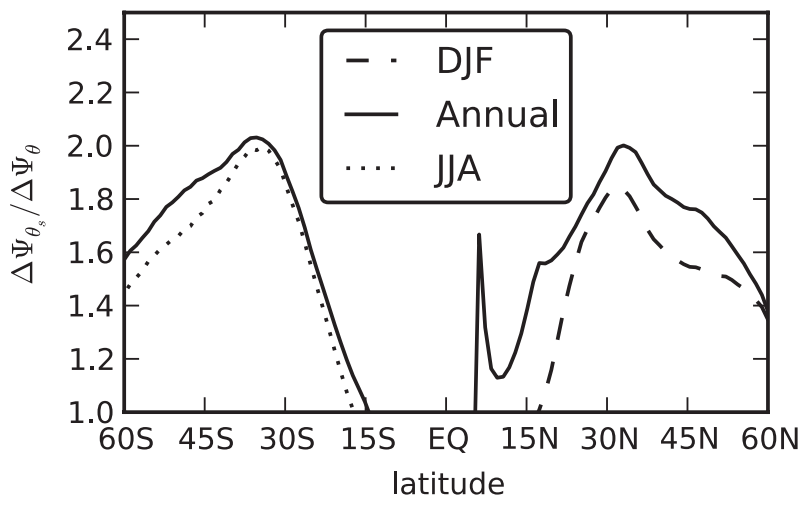

FIG. 4. Ratio of moist circulation to dry circulation in ERA-40. Summertime (NH: JJA; SH: DJF) ratios are not shown because the assumptions behind the theory presented in this paper are not expected to hold.

In the model derived here, $\Delta p_{s}$ is related to the standard deviation of surface dry potential temperature through $\Delta p_{s}=g H \Delta \theta_{s}$. If we assume that the lowertropospheric bulk stratification $H$ is constant, then a larger surface temperature variability would lead to a larger $\Delta p_{s}$. The midlatitude annual-mean temperature variability at the earth's surface being larger than the wintertime variability in both hemispheres, the annualmean $\Delta p_{s}$ should be larger than the wintertime $\Delta p_{s}$. For a fixed mixed layer depth $\Delta p_{m}$, the annual-mean $\Delta p_{s} / \Delta p_{m}$ would thus be larger than the wintertime $\Delta p_{s} / \Delta p_{m}$ and, consequently, the annual-mean circulation ratio should be larger than the wintertime circulation ratio, as confirmed by Fig. 4 .

With our model, we can investigate how the relative contribution of the moist and dry circulations would change in different climates. In wintertime and for both hemispheres, an analysis of phase 3 of the Coupled Model Intercomparison Model (CMIP3) simulations indicates that the moist circulation should strengthen relative to the dry circulation over the next centuries (Laliberté and Pauluis 2010). In those simulations, the wintertime moist circulation does not change significantly in $\mathrm{NH}$ and it weakens slightly in the $\mathrm{SH}$. We can explain this by noting that the observed $\mathrm{SH}$ wintertime ratio of circulation strengths peaks at around 2 while the observed $\mathrm{NH}$ wintertime ratio of circulation strengths peaks at around 1.8. In the context of our theory, we conclude that the $\mathrm{SH}$ wintertime meridional circulation has reached its maximum ratio of circulation strengths while the $\mathrm{NH}$ wintertime meridional circulation has the possibility become even more dominated by the moist circulation. This "saturation" of the overturning circulation in warm climates has been previously observed in modeling studies by Caballero and Langen (2005), 
O'Gorman and Schneider (2008), and Rose and Ferreira (2013).

\section{b. Meridional heat fluxes}

As explained in the introduction, the dry and moist isentropic circulations are directly related to sensible and total meridional heat fluxes, respectively. The change in strength of the two circulations in response to global warming reflects a change in the relative contribution of sensible heat fluxes to the total heat fluxes. Laliberté and Pauluis (2010) showed that, across all seasons in CMIP3 simulations, meridional sensible heat fluxes weakened and total meridional heat fluxes strengthened. The total meridional heat fluxes become increasingly dominated by latent heat fluxes under that climate change scenario. This suggests that since the relative strength of the moist and dry circulations "saturate" in a warming climate, the relative contribution of the sensible meridional heat fluxes to the total-sensible plus latent-meridional heat fluxes is likely to saturate as well. This is consistent with the results of Caballero and Langen (2005). To investigate this question using our model, we must first derive an expression for the ratio of the meridional entropy fluxes.

The column-integrated dry meridional entropy fluxes $F_{\theta}$ can be obtained from the dry circulation strength $\Delta \Psi_{\theta}$ and from the difference in potential temperature between the poleward and equatorward branches of the circulation $\left(\theta^{+}-\theta^{-}\right)$:

$$
F_{\theta}=\left(\theta^{+}-\theta^{-}\right) \Delta \Psi_{\theta}
$$

where $^{1}$

$$
\begin{aligned}
& \theta^{+}=\int_{0}^{\infty} \theta \max \left(\partial_{\theta} \Psi_{\theta}, 0\right) d \theta / \int_{0}^{\infty} \max \left(\partial_{\theta} \Psi_{\theta}, 0\right) d \theta, \\
& \theta^{-}=\int_{0}^{\infty} \theta \min \left(\partial_{\theta} \Psi_{\theta}, 0\right) d \theta / \int_{0}^{\infty} \min \left(\partial_{\theta} \Psi_{\theta}, 0\right) d \theta .
\end{aligned}
$$

Figure 5 (bottom) shows the mean potential temperature in the poleward and equatorward branches of the circulations as thick gray lines and the mean surface

\footnotetext{
${ }^{1}$ In these computations, we have set $\Psi_{\theta}, \Psi_{\theta_{e}}=0$ where their magnitude was less than $10 \mathrm{~Sv}\left(1 \mathrm{~Sv} \equiv 10^{6} \mathrm{~m}^{3} \mathrm{~s}^{-1}\right)$. This eliminates the upper-tropospheric contributions from the descending branch of the Hadley cell and thus focuses our attention on lower- to midtropospheric processes.
}
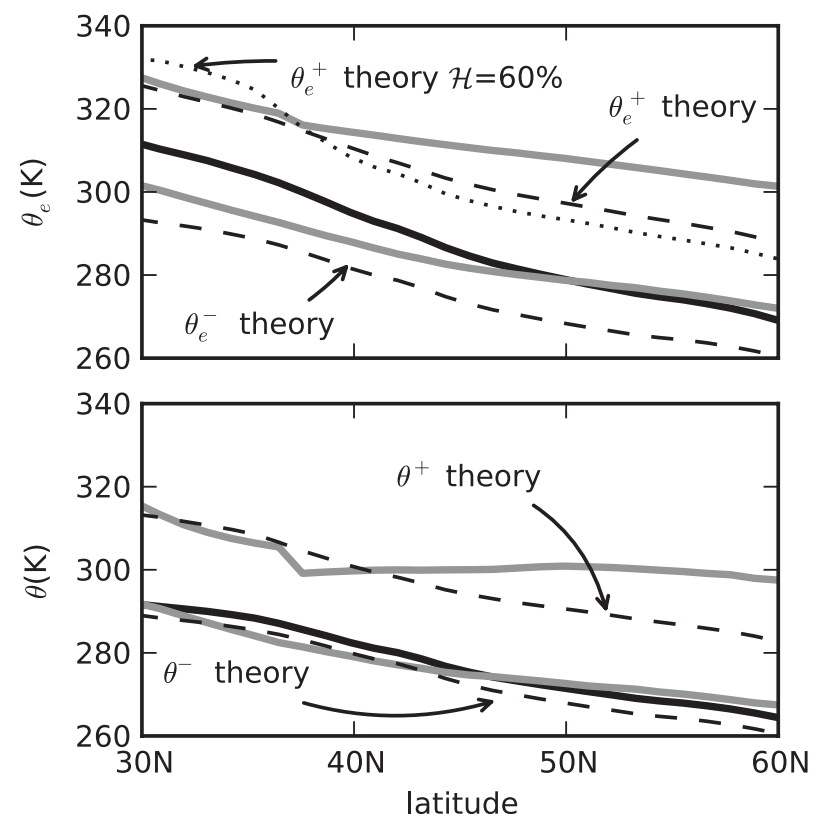

FIG. 5. (top) The thick black line shows $\overline{\theta_{e s}}$ from observations. Thick gray lines show the typical $\theta_{e}^{+}$(upper line) and $\theta_{e}^{-}$(lower line) from observations. Dashed lines show $\theta_{e}^{-}$and $\theta_{e}^{+}$from Eqs. (14b) and (14c), respectively, with $\overline{\theta_{e s}}$ and $\Delta \theta_{e s}$ taken from observations. The dotted line shows $\theta_{e}^{+}$computed from the linearization in Eq. (9a) and the observations of $\overline{\theta_{s}}$ and $\Delta \theta_{s}$. (bottom) As at (top), but for the dry circulation. The dashed lines are computed from Eqs. (14a) and (14d). For both panels, we used $\Delta p_{s} / \Delta p_{m}=1$.

potential temperature is shown as a thick black line. The moist versions of Eqs. (12) and (13) are obtained by replacing $\theta$ by $\theta_{e}$ and are shown in Fig. 5 (top).

The typical dry potential temperature $\theta^{-}$in the equatorward branch of the dry circulation is very close to $\overline{\theta_{s}}$ over the whole hemisphere. For the typical equatorward equivalent potential temperature $\theta_{e}^{-}$, the same is true poleward of $45^{\circ} \mathrm{N}$. Between $30^{\circ}$ and $40^{\circ} \mathrm{N}, \overline{\theta_{e s}}$ is about halfway between $\theta_{e}^{+}$and $\theta_{e}^{-}$, indicating that the moist circulation is centered around surface values in that region.

The entropy fluxes from our theoretical model can be obtained in a similar way. We first obtain expressions for $\theta_{e}^{+}, \theta_{e}^{-}, \theta^{+}$, and $\theta^{-}$using our model. The typical potential temperatures in the equatorward branches of the moist and dry circulations are obtained from Eqs. (9) by assuming that all equatorward flows occur solely in the surface and mixed layer:

$$
\begin{aligned}
\theta^{-} \text {theory } & =\int_{0}^{\infty} \theta\langle\rho v\rangle^{-} d \theta / \int_{0}^{\infty}\langle\rho v\rangle^{-} d \theta, \\
& \approx \overline{\theta_{s}}-1.25 \Delta \theta_{s} /\left\{1+2.5\left(\Delta p_{s} / \Delta p_{m}\right)\right\},
\end{aligned}
$$




$$
\begin{aligned}
\theta_{e}^{-} \text {theory }= & \int_{0}^{\infty} \theta_{e m}(\theta) \min \left\{\langle\rho v\rangle_{\mathrm{m}}, 0\right\}+\cdots \\
& \theta\langle\rho v\rangle_{\mathrm{s}} d \theta / \int_{0}^{\infty}\langle\rho v\rangle^{-} d \theta \\
\approx & \frac{\left\{\overline{\theta_{e s}}+2.5\left(\Delta p_{s} / \Delta p_{m}\right) \overline{\theta_{s}}\right\}-1.25 \Delta \theta_{e s}}{1+2.5\left(\Delta p_{s} / \Delta p_{m}\right)},
\end{aligned}
$$

where $\Delta \theta_{e s}=L \Delta \theta_{s}$.

The expression for the typical equivalent potential temperature in the poleward branch of the circulation $\theta_{e}^{+}$can be found by assuming that all of the moisture transport in the lower troposphere occurs within the mixed layer and that $\theta_{e}$ remains constant for air parcels that undergo large-scale moist ascent within midlatitude eddies. The result is a $\theta_{e}^{+}$equal to the typical $\theta_{e}$ in the poleward branch of the mixed-layer mass fluxes and, as a consequence, it is directly related to the variance of the Gaussian distribution:

$$
\begin{aligned}
\theta_{e}^{+} \text {theory } & =\int_{0}^{\infty} \theta_{e m}(\theta)\langle\rho v\rangle^{+} d \theta / \int_{0}^{\infty}\langle\rho v\rangle^{+} d \theta, \\
& \approx \overline{\theta_{e s}}+1.25 \Delta \theta_{e s},
\end{aligned}
$$

where $\Delta \theta_{e s}=L \Delta \theta_{s}$. This result is similar to Pauluis et al. (2011) where variances of $\theta_{e}$ in the planetary boundary layer were related to the bulk atmospheric stratification.

It was observed in LSP that poleward-moving air parcels follow a moist isentrope, either through upright or slantwise convection. It is thus expected that moist lower-tropospheric air masses, once they saturate, will ascend to dry potential temperatures similar to their initial equivalent potential temperatures. These moist ascents give rise to a moist recirculation whose vertical extent in $\theta$ is related to lower-tropospheric values of $\theta_{e}$ in the poleward flows. This observation suggests that $\theta^{+}$, the typical potential temperature in the poleward branch of the dry circulation, occurs within the moist recirculation. In Fig. 1, we see that $\theta^{+}$is found approximately at the midpoint along a vertical line between the peak of the poleward mixed-layer mass fluxes $(\theta \approx$ $\left.292 \mathrm{~K}, \theta_{e} \approx 315 \mathrm{~K}\right)$ and its intersection with the main diagonal $\left(\theta=\theta_{e} \approx 315 \mathrm{~K}\right)$. At the peak of the poleward mixed-layer mass fluxes, the equivalent potential temperature is given by Eq. (14c) and the dry potential temperature can be found in a similar way. This indicates that $\theta^{+}$can be approximated by

$$
\theta^{+} \text {theory } \approx 0.5\left(\int_{0}^{\infty} \theta\langle\rho v\rangle^{+} d \theta / \int_{0}^{\infty}\langle\rho v\rangle^{+} d \theta\right)+0.5 \theta_{e}^{+},
$$

$$
\approx 0.5\left(\overline{\theta_{e s}}+1.25 \Delta \theta_{e s}\right)+0.5\left(\overline{\theta_{s}}+1.25 \Delta \theta_{s}\right) .
$$

Note that, as in the circulation strengths ratio, $\alpha$ has disappeared from Eqs. (14). This means that the meridional velocities' magnitudes are not directly determining the potential temperatures in the different branches of the circulations.

In Fig. 5, we compare the mean, poleward, and equatorward potential and equivalent potential temperature from the ERA-40 data to the model predictions in Eqs. (14) during DJF in the NH. The observed $\theta^{+}, \theta^{-}$, $\theta_{e}^{+}$, and $\theta_{e}^{-}$are shown as thick gray lines. In the top panel, we plot as dashed curves Eqs. (14b) and (14c), using $\overline{\theta_{e s}}$ and $\Delta \theta_{e s}$ from observations and with $\Delta p_{s} / \Delta p_{m}=1$. The poleward $\theta_{e}^{+}$and equatorward $\theta_{e}^{-}$from the theory are closest to observations between $30^{\circ}$ and $40^{\circ} \mathrm{N}$, with a cold bias of approximately 2 and $5 \mathrm{~K}$, respectively. This bias in $\theta_{e}^{-}$is a consequence of our theory not accounting for some of the equatorward mass fluxes occurring in the free troposphere-fluxes that are associated with the moist recirculation. In the same panel, we used a dotted curve to plot Eq. (14c) with $\overline{\theta_{e s}}$ and $\Delta \theta_{e s}$ computed from $\overline{\theta_{s}}$ and $\Delta \theta_{s}$ using the linearization in Eqs. (9) with $60 \%$ relative humidity. This time, the theory is biased warm between $30^{\circ}$ and $38^{\circ} \mathrm{N}$, by about $5 \mathrm{~K}$. This is likely due, among other things, to the neglect of pressure differences in the linearization equations (9).

In the bottom panel of Fig. 5, the dashed curves show $\theta^{+}$and $\theta^{-}$from the theory. The predictions from the theory are within approximately $1 \mathrm{~K}$ of observations between $30^{\circ}$ and $40^{\circ} \mathrm{N}$. The theoretical model predictions in Eqs. (14) capture the observations reasonably well between $30^{\circ}$ and $40^{\circ} \mathrm{N}$, where the isentropic circulations are the strongest. A detailed understanding of why there is such a good agreement with observations for $\theta^{+}$is beyond the scope of this paper.

With Eq. (11) and the typical potential temperatures in Eqs. (14), we can now obtain an expression for the column-integrated moist entropy fluxes relative to the column-integrated dry entropy fluxes:

$$
\frac{F_{\theta_{e}}}{F_{\theta}}=\left(\frac{\theta_{e}^{+}-\theta_{e}^{-}}{\theta^{+}-\theta}\right) \frac{\Delta \Psi_{\theta_{e}}}{\Delta \Psi_{\theta}} .
$$

Unlike the formula for the relative strengths of the circulations [Eq. (11)], this expression will depend on $\Delta p_{s} / \Delta p_{m}$ as well as on the statistics of the surface potential temperature distribution. Using statistics at $35^{\circ} \mathrm{N}$ during DJF, we plot Eq. (15) in Fig. 3. The resulting curve has a shape similar to $\Delta \Psi_{\theta_{e}} / \Delta \Psi_{\theta}$ except that it asymptotically approaches a ratio of 2.8 for large $\Delta p_{s} / \Delta p_{m}$. This is 
in broad agreement with the maximum ratio of meridional heat fluxes that can be inferred from results by Caballero and Langen (2005) and Rose and Ferreira (2013).

In a warming world with a nearly constant surface relative humidity [as suggested by Schneider et al. (2010)], the linear factor relating the variance of equivalent potential temperature to the variance of dry potential temperature in Eq. (9a) will increase. This effect will make the maximum attainable by the ratio $\left(\theta_{e}^{+}-\theta_{e}^{-}\right) /\left(\theta^{+}-\theta^{-}\right)$even larger in warmer climates. It, however, does not change the fact that, in the context of the theory presented here, the ratio of moist to dry entropy fluxes will saturate at large $\Delta p_{s} / \Delta p_{m}$.

Using Eq. (15) we can gain some insight into why the ratio $F_{\theta_{e}} / F_{\theta}$ saturates at higher values than $\Delta \Psi_{\theta_{e}} / \Delta \Psi_{\theta}$. The ratio $\left(\theta_{e}^{+}-\theta_{e}^{-}\right) /\left(\theta^{+}-\theta^{-}\right)$is larger than unity because, as it can be seen from Eq. (14b), $\theta_{e}^{-}$is a mixture of moist mixed-layer air and dry surface-layer air. It is therefore less than the typical equivalent potential temperature in the equatorward flow of the mixed layer. As $\Delta p_{s} / \Delta p_{m}$ increases, the mixed-layer contribution to the total equatorward fluxes is reduced, making $\theta_{e}^{-}$ increasingly dry until it becomes equal to $\overline{\theta_{s}}$ for large $\Delta p_{s} / \Delta p_{m}$. This is the same value attained by $\theta^{-}$in that limiting case. The ratio $\left(\theta_{e}^{+}-\theta_{e}^{-}\right) /\left(\theta^{+}-\theta^{-}\right)$thus increases with increasing $\Delta p_{s} / \Delta p_{m}$ because the equatorward flow from the surface layer "dries" the net return flow of the circulation while it does not affect the moisture content of the poleward flow. Equation (15) could also be interpreted over a range of parameters $\overline{\theta_{s}}$ and $\Delta \theta_{s}$ to cover a parameter space similar to Caballero and Langen (2005) and Rose and Ferreira (2013).

\section{Summary and discussion}

A theoretical model describing the near-surface flow of the dry and moist isentropic circulations was derived. This model represents an extension of the dry model of Held and Schneider (1999) and uses the surface potential temperature distribution. The model decomposes the low-level flow into contributions from a near-surface mixed layer and a stratified layer right above it. Reanalysis data suggest that the low-level flow can be decomposed into three main components with distinct thermodynamic characteristics: 1 ) an equatorward flow of cold, moist air at values of $\theta$ less than the mean surface temperature $\overline{\theta_{s}} ; 2$ ) an equatorward flow of dry air in the stratified layer at values of $\theta$ higher than $\overline{\theta_{s}}$; and 3 ) a poleward flow of warm, moist air within the mixed layer, with $\theta>\overline{\theta_{s}}$.

In this work, we used the model to understand how the differences between the dry and the moist circulations might evolve in different climates. Since differences in the two circulations are resulting from large-scale moist ascents in midlatitude eddies (Laliberté et al. 2012), changes in surface thermodynamics are expected to influence the balance between the two circulations. Indeed, when the surface layer deepens owing to an increase in surface temperature variability, this expression constrains the ratio of the moist to dry circulation strengths to be at most 2. For shallower surface layers typical of winter, it instead indicates a ratio between 1.5 and 2. As the annual-mean circulation is already near the extreme case of the idealized model, the ratio between the dry and moist circulation is likely to remain unchanged. During the winter, by contrast, an increase in the surface layer depth would lead to a strengthening of the moist circulation relative to the dry circulation. Circulation strength ratios from ERA-40 are in qualitative agreement with these results.

A relative intensification of the moist circulation would increase the fraction of latent heat fluxes in the total (sensible plus latent) meridional heat fluxes. Using our model, we quantified this relationship between the overturning circulations and the meridional heat fluxes by first obtaining expressions for the typical potential temperatures in the poleward and equatorward branches of the moist and dry circulations [Eq. (14)] using the distribution of potential temperature at the earth's surface. A comparison with ERA-40 during DJF in the $\mathrm{NH}$ shows that these typical potential temperatures are in good agreement with observations between $30^{\circ}$ and $40^{\circ} \mathrm{N}$. Under a Gaussian distribution assumption, we have shown that the equivalent potential temperature in the poleward flow is equal to its mean value plus 1.25 times its standard deviation at the earth's surface. In effect, if we consider that moist air parcels ascend adiabatically, this implies that the midlatitude dry stratification would be affected by changes in the low-level variance of moist air, as noted by Juckes (2000), Frierson (2006), and Pauluis et al. (2011). In a warming climate where temperature, moisture, and their variances at the earth's surface are expected to change, it could lead to considerable impacts on the mean overturning circulation, the hydrological cycle, and the meridional heat fluxes.

The effect on the meridional heat fluxes was investigated by combining the ratio of circulation strengths with the typical potential temperatures in the poleward and equatorward branches of the circulations to obtain an expression [Eq. (15)] for the ratio of total heat fluxes to sensible heat fluxes. This expression showed that, under an increasing surface-layer depth, the ratio of heat fluxes exhibited a behavior similar to the ratio of circulation strengths. Specifically, using fixed surface 
temperatures obtained from observations during DJF at $35^{\circ} \mathrm{N}$, it is constrained to be less than 2.8 and it attains its maximum for a surface layer deeper than the one needed for the ratio of circulation strengths to attain its maximum. We concluded that this behavior supports the results of Caballero and Langen (2005) where it can be observed that while the meridional overturning circulation might weaken, the total meridional heat fluxes can strengthen in warming climates.

These effects have also been observed by Laliberté and Pauluis (2010) in global warming simulations from the CMIP3 archive. The model described here captures some of the dynamics of the response to climate change and therefore highlights the role of surface moist thermodynamics in setting the midlatitude overturning circulation. In Laliberté and Pauluis (2010), it was explained that the difference between the moist and dry circulations in the winter hemispheres would increase, implying a winter overturning circulation increasingly made up of motions undergoing large-scale saturated ascent. A strengthened overturning implies an intensified hydrological cycle during the winter months, which could potentially lead to stronger storms accompanied by changing precipitation patterns; hence, it appears necessary to constrain changes in the circulation to improve the reliability of future climate change predictions.

Acknowledgments. We thank the Computational and Information Systems Laboratory at the National Center for Atmospheric Research for providing the ECMWF data. Frédéric Laliberté was supported by a McCracken Fellowship from the New York University and by the NSERC/CRSNG doctoral fellowship program. This work was supported by the NSF under Grant AGS0944058. The authors are also grateful to Brian Rose and Aaron Donohue whose comments greatly improved the manuscript.

\section{APPENDIX}

\section{Mathematical Identities for a Gaussian Probability Distribution}

In the following expression, $\Pi(\theta)$ is a Gaussian probability distribution function with mean $\overline{\theta_{s}}$ and variance $\left(\Delta \theta_{s}\right)^{2}$. It is assumed that $\overline{\theta_{s}}$ is several standard deviations away from zero:

$$
\begin{aligned}
\int_{0}^{\theta}\left(\theta^{\prime}-\overline{\theta_{s}}\right) \Pi\left(\theta^{\prime}\right) d \theta^{\prime} & =-\left(\Delta \theta_{s}\right)^{2} \int_{0}^{\theta} \frac{d \Pi\left(\theta^{\prime}\right)}{d \theta^{\prime}} d \theta^{\prime} \\
& =-\left(\Delta \theta_{s}\right)^{2} \Pi(\theta),
\end{aligned}
$$

$$
\begin{gathered}
\int_{0}^{\infty} \max \left(\overline{\theta_{s}}-\theta, 0\right) \Pi(\theta) d \theta=-\left(\Delta \theta_{s}\right)^{2} \int_{\overline{\theta_{s}}}^{\infty} \frac{d \Pi\left(\theta^{\prime}\right)}{d \theta^{\prime}} d \theta \\
=\Delta \theta_{s} / \sqrt{2 \pi} \\
\int_{0}^{\infty}\left(\theta-\overline{\theta_{s}}\right) \max \left(\theta-\overline{\theta_{s}}, 0\right) \Pi(\theta) d \theta \\
=1 / 2 \int_{-\infty}^{\infty}\left(\theta-\overline{\theta_{s}}\right)^{2} \Pi(\theta) d \theta=\Delta \theta_{s}^{2} / 2 .
\end{gathered}
$$

In this paper, we will use the approximation $\sqrt{2 \pi} \approx 2.5$ in order to simplify the discussion.

The derivation of the model assumes a Gaussian distribution of temperature at the earth's surface. To assess the validity of the Gaussian assumption, we compute its error $E$ by using

$$
E=\int_{0}^{\overline{\theta_{s}}}\left[\Pi\left(\theta^{\prime}\right)+\left(\Delta \theta_{s}\right)^{-2} \int_{0}^{\theta^{\prime}}\left(\theta^{\prime \prime}-\overline{\theta_{s}}\right) \Pi\left(\theta^{\prime \prime}\right) d \theta^{\prime \prime}\right] d \theta^{\prime} .
$$

This nondimensional expression gives the error in computing the surface layer contribution to $\Delta \Psi_{\theta}$ in Eq. (11) as a fraction of the total mass flux in the surface layer. This error is less than $5 \%$ in the midlatitudes for the annual mean, less than $5 \%$ in SH for JJA, and less than $20 \%$ in NH for DJF.

\section{REFERENCES}

Caballero, R., and P. L. Langen, 2005: The dynamic range of poleward energy transport in an atmospheric general circulation model. Geophys. Res. Lett., 32, L02705, doi:10.1029/ 2004GL021581.

Czaja, A., and N. Blunt, 2011: A new mechanism for oceanatmosphere coupling in midlatitudes. Quart. J. Roy. Meteor. Soc., 137, 1095-1101.

Eckhardt, S., A. Stohl, H. Wernli, P. James, C. Forster, and N. Spichtinger, 2004: A 15-year climatology of warm conveyor belts. J. Climate, 17, 218-237.

Emanuel, K., 1994: Atmospheric Convection. Oxford University Press, $580 \mathrm{pp}$.

Frierson, D. M. W., 2006: Robust increases in midlatitude static stability in simulations of global warming. Geophys. Res. Lett., 33, L24816, doi:10.1029/2006GL027504.

Held, I., and T. Schneider, 1999: The surface branch of the zonally averaged mass transport circulation in the troposphere. J. Atmos. Sci., 56, 1688-1697.

Juckes, M. N., 2000: The static stability of the midlatitude troposphere: The relevance of moisture. J. Atmos. Sci., 57, 3050-3057.

Laliberté, F., and O. Pauluis, 2010: Winter intensification of the moist branch of the circulation in simulations of 21st century climate. Geophys. Res. Lett., 37, L20707, doi:10.1029/ 2010 GL045007.

— T. Shaw, and O. Pauluis, 2012: Moist recirculation and water vapor transport on dry isentropes. J. Atmos. Sci., 69, 875-890. 
O'Gorman, P., and T. Schneider, 2008: Energy of midlatitude transient eddies in idealized simulations of changed climates. J. Climate, 21, 5797-5806.

Pauluis, O., A. Czaja, and R. Korty, 2008: The global atmospheric circulation on moist isentropes. Science, 321, 1075-1078.

, — - and — 2010: The global atmospheric circulation in moist isentropic coordinates. J. Climate, 23, 3077-3093.

__ T. Thaw, and F. Laliberté, 2011: A statistical generalization of the transformed Eulerian-mean circulation for an arbitrary vertical coordinate system. J. Atmos. Sci., 68, 17661783.
Rose, B. E. J., and D. Ferreira, 2013: Ocean heat transport and water vapor greenhouse in a warm equable climate: A new look at the low gradient paradox. J. Climate, in press.

Schneider, T., K. Smith, P. O'Gorman, and C. Walker, 2006: A climatology of tropospheric zonal-mean water vapor fields and fluxes in isentropic coordinates. J. Climate, 19, 5918-5933.

— P. O'Gorman, and X. Levine, 2010: Water vapor and the dynamics of climate changes. Rev. Geophys., 48, RG3001, doi:10.1029/2009RG000302.

Uppala, S. M., and Coauthors, 2005: The ERA-40 Re-Analysis. Quart. J. Roy. Meteor. Soc., 131, 2961-3012. 